\title{
Potential Risks of Pharmacy Compounding
}

\author{
Jennifer Gudeman • Michael Jozwiakowski • \\ John Chollet $\cdot$ Michael Randell
}

Published online: 23 March 2013

(C) The Author(s) 2013. This article is published with open access at Springerlink.com

\begin{abstract}
Pharmacy compounding involves the preparation of customized medications that are not commercially available for individual patients with specialized medical needs. Traditional pharmacy compounding is appropriate when done on a small scale by pharmacists who prepare the medication based on an individual prescription. However, the regulatory oversight of pharmacy compounding is significantly less rigorous than that required for Food and Drug Administration (FDA)-approved drugs; as such, compounded drugs may pose additional risks to patients. FDA-approved drugs are made and tested in accordance with good manufacturing practice regulations (GMPs), which are federal statutes that govern the production and testing of pharmaceutical products. In contrast, compounded drugs are exempt from GMPs, and testing to assess product quality is inconsistent. Unlike FDAapproved drugs, pharmacy-compounded products are not clinically evaluated for safety or efficacy. In addition, compounded preparations do not have standard product labeling or prescribing information with instructions for safe use. Compounding pharmacies are not required to report adverse events to the FDA, which is mandatory for manufacturers of FDA-regulated medications. Some pharmacies engage in activities that extend beyond the boundaries of traditional pharmacy compounding, such as large-scale production of compounded medications without individual patient prescriptions, compounding drugs that
\end{abstract}

J. Gudeman · M. Jozwiakowski · J. Chollet $(\bowtie)$

Ther-Rx Corporation, 2280 Schuetz Road, St. Louis, MO 63146, USA

e-mail: jchollet@ther-rx.com

M. Randell

Northside Hospital, Atlanta, GA, USA have not been approved for use in the US, and creating copies of FDA-approved drugs. Compounding drugs in the absence of GMPs increases the potential for preparation errors. When compounding is performed on a large scale, such errors may adversely affect many patients. Published reports of independent testing by the FDA, state agencies, and others consistently show that compounded drugs fail to meet specifications at a considerably higher rate than FDAapproved drugs. Compounded sterile preparations pose the additional risk of microbial contamination to patients. In the last 11 years, three separate meningitis outbreaks have been traced to purportedly 'sterile' steroid injections contaminated with fungus or bacteria, which were made by compounding pharmacies. The most recent 2012 outbreak has resulted in intense scrutiny of pharmacy compounding practices and increased recognition of the need to ensure that compounding is limited to appropriate circumstances. Patients and healthcare practitioners need to be aware that compounded drugs are not the same as generic drugs, which are approved by the FDA. The risk-benefit ratio of using traditionally compounded medicines is favorable for patients who require specialized medications that are not commercially available, as they would otherwise not have access to suitable treatment. However, if an FDA-approved drug is commercially available, the use of an unapproved compounded drug confers additional risk with no commensurate benefit.

\section{Introduction}

A brand name drug is a prescription medication that has been approved by the Food and Drug Administration (FDA) based on comprehensive toxicological data and human clinical trials demonstrating that the drug is safe and 
effective, and chemistry evaluations proving that the product can be made consistently to a high quality standard. After the patent protection period of the branded drug expires, the FDA may approve generic drugs that have been tested and confirmed to be bioequivalent to the brand name product.

Pharmacy compounding of individualized medicines is necessary when an FDA-approved drug product is not available or appropriate for the patient, or must be altered in some manner, such as strength or route of delivery. Traditional pharmacy compounding provides a valuable service that is an essential element of our healthcare system.

FDA-approved drugs-branded and generic alike-are manufactured under good manufacturing practice regulations (GMPs), which are federal statutes that govern the production and testing of pharmaceutical materials. The FDA regulates and regularly inspects pharmaceutical manufacturing facilities to ensure compliance with GMPs. In contrast, pharmacies are primarily under the authority of state Boards of Pharmacy, whose regulations may incorporate some or all of United States Pharmacopeia (USP) chapters $\langle 795\rangle$ Pharmaceutical Compounding-Nonsterile Preparations and $\langle 797\rangle$ Pharmaceutical CompoundingSterile Preparations. Pharmacies are exempt from GMP regulations and only undergo FDA inspections in rare instances. As a result, there is less assurance of consistent quality for compounded preparations than there is for FDAapproved drugs [1-3].

Current events involving compounding pharmacies highlight the need for greater understanding of the differences between FDA-approved drugs and pharmacy-compounded preparations. In 2011, the American College of Obstetricians and Gynecologists (ACOG) stated that healthcare providers should understand the inherent differences between an FDA-approved manufactured product and a compounded preparation [4]. A recent paper in the Journal of the American Medical Association states that physicians and patients should discuss the potential risks when prescribing compounded products [5].

\section{FDA-Approved Drugs and GMPs}

Under the Federal Food, Drug, and Cosmetic Act, brand name drugs and generic drugs approved by the FDA must be safe and effective, and must be manufactured in accordance with current GMPs to ensure their identity, strength, quality, and purity [6]. GMPs are legally enforceable regulations that specify how pharmaceutical manufacturing, packaging, labeling, testing, and distribution must be done for FDA-approved products manufactured domestically or imported into the US. The FDA performs regular inspections of all facilities manufacturing FDA-approved drugs, both in the US and abroad, to ensure compliance with GMPs.

GMPs include provisions for the facilities and equipment used to manufacture drugs, the education and training of personnel, and the calibration and cleaning of process equipment. Validated analytical test procedures are used to ensure that drugs conform to FDA-approved specifications for potency, purity, and other requirements such as sterility. All incoming ingredients and components must be retested upon receipt, and manufacturing processes must be validated to consistently meet quality standards. GMPs also require an independent quality control unit to oversee the manufacturing, packaging, and testing processes and to reject substandard batches. Stability studies must be performed to support expiration dating of products.

\section{Pharmacy Compounding}

\subsection{Traditional Pharmacy Compounding}

The FDA defines traditional pharmacy compounding as the combining, mixing, or altering of ingredients to create a customized medication for an individual patient in response to a licensed practitioner's prescription [1]. The National Association of Boards of Pharmacy (NABP) further describes compounding as the result of a practitioner's prescription drug order based on the practitioner/patient/ pharmacist relationship in the course of professional practice [7]. Traditional pharmacy compounding plays a valuable role in providing access to medications for individuals with unique medical needs, which cannot be met with a commercially available product. For instance, a prescriber may request that a pharmacist compound a suspension for a pediatric or geriatric patient unable to swallow a medication in its commercially available form. In traditional pharmacy compounding, an individualized medicine is prepared at the request of a prescriber on a small scale.

\subsection{Non-Traditional Pharmacy Compounding}

Some pharmacies have seized upon a burgeoning business opportunity to expand their activities beyond the scope of traditional pharmacy compounding [8]. Examples of improper pharmacy compounding include introducing drug moieties that have not been approved for use in the US or have been removed by the FDA for safety reasons, largescale production of compounded medications without prescriptions, and creating copies (or essentially copies) of FDA-approved drugs. 
The FDA issued letters in 2004 to compounding pharmacies obtaining domperidone from foreign sources for women to assist with lactation, noting that domperidone is not approved in the US for any indication. Citing public health risks, including cardiac arrest and sudden death, the FDA recommended that breastfeeding women avoid the use of domperidone [9].

The FDA has publically expressed concerns regarding "large-scale drug manufacturing under the guise of pharmacy compounding" $[1,2]$. The FDA has noted that poor practices on the part of drug compounders can result in contamination or in products that do not possess the strength, quality, and purity required [2]. Unlike FDA-approved products, consumers and prescribers cannot assume that compounded drugs were made by validated processes in properly calibrated and cleaned equipment; that the ingredients in the drug were obtained from FDA-approved sources; that production personnel had the requisite knowledge and training; and that appropriate laboratory testing was performed to verify the compounded drug's potency, purity, and quality. In the case of sterile compounding, there are also concerns about the adequacy of environmental monitoring, which includes microbiological testing of the facility, equipment, air purification, and water. The shelf-life of compounded products is typically not verified by stability testing; therefore, compounded preparations cannot be assumed to retain their original strength and purity over time.

Pharmacies making copies of commercially available products for economically driven reasons, rather than genuine medical need, are also engaged in improper compounding, as this circumvents important public health requirements [10]. A significant concern is the use of active and inactive ingredients that are from foreign sources and not manufactured under GMPs to create the unapproved copies. The FDA has stated that consumers would be better served by commercially available drugs, which have been determined to be safe and effective and manufactured under rigorous GMP requirements [1].

In 2001, a Kansas City-based pharmacist was discovered to have adulterated 72 different drugs, including many oncology medications, to increase profits. According to law enforcement estimates, the pharmacist diluted approximately 98,000 prescriptions for 4,200 patients over an 11-year time period [11]. This drug adulteration was detected not by clinicians or patients, but rather by a pharmaceutical sales representative who noted that the pharmacy was selling considerably more drugs than it was buying. Illegal activities of this nature are by no means typical of pharmacy compounding, but this case illustrates that clinical observation alone cannot be relied upon to detect quality problems in medicines.

\subsection{Compounded Sterile Preparations (CSPs)}

The primary standard for the compounding of sterile medications is USP chapter $\langle 797\rangle$ Pharmaceutical Compounding: Sterile Preparations, which specifies the conditions and practices that should be used to prevent harm to patients from microbial contamination, bacterial endotoxins, chemical and physical contaminants, and ingredients of inappropriate quality. USP $\langle 797\rangle$ classifies aseptic manipulation of sterile products or ingredients as low-risk sterile compounding. However, the sterility assurance level (SAL) of preparations compounded by an aseptic process is, at best, several orders of magnitude lower than the SAL of terminally sterilized pharmaceutical products manufactured under GMPs. The SAL is much lower still if the aseptic compounding process has not been robustly validated [12]. Incorporating non-sterile ingredients into a compounded preparation prior to terminal sterilization is classified as high-risk sterile compounding [13]. USP $\langle 797\rangle$ states that high-risk CSPs should be used within $24 \mathrm{~h}$ of preparation if stored at room temperature, or 3 days if refrigerated, unless sterility testing is conducted to support extended dating. USP chapter $\langle 71\rangle$ Sterility Tests emphasizes that sterility tests are not by themselves designed to ensure that a batch of product is sterile; rather, this is primarily accomplished by validation of the sterilization process [14].

By law, USP $\langle 797\rangle$ is enforceable by the FDA, but in practice the agency generally defers regulation of pharmacies to states [8]. The NABP has incorporated USP $\langle 797\rangle$ into its Model State Pharmacy Act and Model Rules. Although some states have adopted USP $\langle 797\rangle$ in its entirety, most State Boards of Pharmacy have only incorporated selected portions of USP $\langle 797\rangle$ into their regulations or board policies [15]. Any requirements that are not adopted are not legally enforceable by the state. For example, in 2010 the Texas State Board of Pharmacy rejected a proposal to require the use of sterile gloves and alcohol by pharmacy personnel compounding sterile preparations, despite this being a specific requirement of USP $\langle 797\rangle$ [16].

A 2011 outbreak of Serratia marcescens bacteremia, which infected 19 patients at six Alabama hospitals, 9 of whom died, was caused by contaminated total parenteral nutrition bags from a compounding pharmacy [17, 18]. As a result of this incident, the Institute of Safe Medication Practices (ISMP) recommended that State Boards of Pharmacy require compounding pharmacies within their state to comply with all aspects of USP $\langle 797\rangle$, and inspect these pharmacies regularly to enforce compliance [19]. ISMP stated, "partial compliance will not even partially protect patients from the risk of infection from contaminated CSPs." ISMP concluded, "Unfortunately, there are too many in healthcare who feel that if it hasn't happened to them, the adverse experiences of others do not apply." 
USP $\langle 797\rangle$ is an appropriate and practical guidance to implement in a pharmacy that invests in the required equipment and training. However, USP $\langle 797\rangle$ does not afford the same degree of sterility assurance for compounded drugs that GMPs provide for FDA-approved sterile products [20]. USP $\langle 797\rangle$ does not provide the necessary protection when compounding expands to mass production of drugs, which requires GMP controls.

\subsection{Comparison of Compounded Drugs with FDA-Approved Drugs}

There are significant differences between compounded drugs and FDA-approved drugs. One important difference is that pharmacy compounded products are not clinically tested for safety and efficacy, nor is bioequivalence testing conducted as is required for generic drugs. The type and extent of quality control testing required for FDA-approved drugs is greater than the testing done on compounded preparations. Compounding pharmacies often rely upon Certificates of Analysis from suppliers rather than retesting incoming bulk ingredients as pharmaceutical manufacturers are required to do by GMPs. Another dissimilarity is that compounding pharmacies are exempt from the federal GMP regulations that are obligatory for all approved pharmaceutical manufacturers. The FDA typically only inspects or takes action against pharmacies after serious health problems occur.

Unlike the product labeling of FDA-approved drugs, the labeling of compounded preparations is neither regulated nor standardized. Thus, compounded medications may be dispensed without any instruction regarding contraindications to use, warnings and precautions, drug interactions, etc. Advertising and promotion of approved drugs is subject to FDA oversight and restriction, including fair balance of safety information. By contrast, compounding pharmacies advertise and promote their products without such oversight and may make unsupported claims of efficacy while failing to mention any potential risks and side effects [21]. In order to ensure that patients and healthcare providers are properly informed, it has been proposed that the labeling on compounded preparations should state that they have not been approved as safe and effective by the FDA [22].

Another major difference is that compounding pharmacies are not required to report adverse events to the FDA, whereas adverse event reporting is mandatory for manufacturers of FDA-regulated medications. Thus, adverse events associated with compounded drugs may be difficult to detect, particularly if the affected patients are widely scattered in different geographic areas.
Although the focus of this article is on drugs produced and used in the US, Canadian regulatory authorities have similarly addressed the issue of pharmacy compounded medications. The "Policy on Manufacturing and Compounding Drug Products in Canada" acknowledges compounding as a legitimate part of medical practice, but says it should not be used as a means to bypass the federal drug review and approval system. The policy also states that compounded products must provide a customized medication, without duplicating an approved drug product [23].

\section{Quality Issues with Compounded Medications}

\subsection{Quality Testing of Compounded Drugs by Regulatory Agencies}

The FDA became aware of 55 product quality problems associated with compounded medicines between 1990 and 2001. The agency therefore conducted a limited survey of 29 different compounded medicines sourced from 12 compounding pharmacies, testing 8 different drugs of various dosage types (oral, injectable, topical, etc.) against established quality standards. Ten out of 29 samples (34\%) failed quality testing, mostly for sub-standard potency ranging from 59 to $89 \%$ of the target dose. By comparison, the FDA noted that the failure rate for over 3,000 FDA-approved commercial products tested from 1996 to 2001 was $<2 \%$ [24]. The FDA conducted a follow-up survey in 2006 and found that 12 of the 36 compounded products (33\%) failed quality testing [25]. Most of the failures were again related to potency, ranging from 68 to $268 \%$ of the labeled dosage. The FDA concluded that the compounding processes used at pharmacies most likely caused the quality failures and reiterated that this rate of failure raises public health concerns for compounded drugs.

Annual testing of randomly selected compounded drugs by the Missouri Board of Pharmacy covering the years 2005-2009 showed failure rates between 11.6 and $25.2 \%$, with potency ranging from 0 to $450 \%$ of the labeled dosage [26]. The Ohio State Board of Pharmacy performed similar testing of compounded drugs in 2007, which found potency results ranging from 27 to $87 \%$ of the labeled dosage and 1,380 doses of fungally contaminated products. Thousands of the purportedly sterile compounded products that were examined had not undergone appropriate sterility testing [27]. Over the period 2008-2010, the Texas State Board of Pharmacy found an overall potency failure rate of $23 \%$ for compounded drugs [28]. 
4.2 Scientific Literature on the Quality of Compounded Drugs

Azarnoff et al. [29] tested compounded nitroglycerin ointments $(84,000$ prescriptions in 2004) and found that $46 \%$ failed basic tests for potency and content uniformity. Similar potency variations were found in compounded diaminopyridine products, with assays ranging from 22 to $125 \%$ of the labeled dosage [30]. Goldman investigated content variability of compounded sodium tetradecyl sulfate solutions and found that compounding pharmacies were using a lower-quality ingredient as a starting material, which produced significant concentrations of a highly toxic contaminant called carbitol [31].

Mahaguna et al. compared the quality of compounded vaginal progesterone suppositories with that of the FDA-approved formulation. Only one of the ten pharmacy-compounded products met the labeled potency specifications. There were also large $\mathrm{pH}$ differences in the suppositories, and the products from one compounding pharmacy were microbially contaminated [32]. An investigation of the quality of compounded hydroxyprogesterone caproate (HPC) samples obtained from 30 compounding pharmacies across the US found that $27 \%$ failed to meet potency standards, and $53 \%$ had impurity levels exceeding those allowed in the FDA-approved version of the drug. Testing of the active pharmaceutical ingredient (API) used to compound the drug product revealed that one sample was glucose, and eight of the other nine API samples exceeded the impurity limits set for HPC used in the FDAapproved drug [33].

A subsequent FDA investigation confirmed instances of variable quality in compounded HPC and the API used to prepare it, which prompted the FDA to remind prescribers and patients that FDA-approved medicines provide a greater assurance of safety and efficacy than compounded drugs [10]. The agency clarified that if there is an FDAapproved drug that is medically appropriate for a patient, the FDA-approved product should be prescribed and used, and reiterated that compounding large volumes of copies of approved drugs does not fall within the scope of traditional pharmacy practice [34].

\section{Adverse Events from Use of Compounded Drugs}

According to the Government Accounting Office, the extent of health problems related to the quality and safety of compounded drugs is unknown, as there is no requirement to report adverse effects of compounded drugs to FDA [35]. Awareness of adverse reactions with compounded medications often originates from media reports of highly noticeable events, such as clusters of infectious outbreaks. Through voluntary reporting, the media, and other sources, the FDA has learned of more than 200 adverse events involving 71 compounded products since 1990 [2]. There are numerous references regarding adverse events associated with the use of compounded products in the scientific literature [27, 36-48].

A 2007 Centers for Disease Control and Prevention (CDC) report described three deaths from cardiac arrest in the Pacific Northwest, which were traced to intravenous colchicine compounded by a pharmacy in Texas [47]. Subsequent investigation found that the compounded preparation contained $4 \mathrm{mg} / \mathrm{mL}$ of colchicine rather than the labeled $0.5 \mathrm{mg} / \mathrm{mL}$ dose. The compounded colchicine injection was subsequently recalled throughout the US.

In August 2011, the FDA issued an alert notifying healthcare providers that repackaged intravitreal injections of bevacizumab used off-label to treat macular degeneration had caused a cluster of eye infections in Florida [45]. Investigators traced Streptococcus infections from multiple eye clinics to one pharmacy, which dispensed the preservative-free product in single-use syringes. Twelve patients were infected, and some lost all of their remaining vision. A later article cited five more patients being blinded in the Los Angeles area, and four patients in Nashville acquired similar infections from the compounded version $[49,50]$.

In September 2012, a cluster of patients in Tennessee contracted fungal meningitis several weeks after receiving an epidural injection of methylprednisolone acetate, which had been compounded by the New England Compounding Center (NECC) in Massachusetts. The CDC estimated the steroid had been injected into roughly 14,000 patients in more than 20 states. Over 500 cases of meningitis were confirmed, and dozens of patients died. Several different fungal species were identified in clinical specimens from the meningitis patients. Testing by the CDC and FDA confirmed the presence of visible contamination and fungus in unopened vials of drug [51]. A subsequent FDA inspection stated that there was no evidence that the process NECC used to sterilize the drugs was effective, and no corrective actions were taken to locate and remove the bacteria and mold from the facility [52].

The 2012 meningitis outbreak was not a unique event. In 2001, five patients were infected with bacterial meningitis, and three died after receiving betamethasone injections contaminated with Serratia bacteria, which had been compounded by a pharmacy in California [53]. In 2002, four women contracted meningitis, and one died, from a steroid injection contaminated with the fungus Exophiala dermatitidis, which had been compounded by a pharmacy in South Carolina [46]. 


\section{Implications for Clinical Practice}

Clinicians and patients rely upon the FDA to ensure that approved drugs have demonstrated safety and efficacy in controlled clinical trials and are manufactured in accordance with federal standards. When there are unique medical needs that cannot be met with commercially available drugs, it may be in a patient's best interests to receive a compounded medication. In such cases, the prescriber should discuss this with the patient, obtain their consent, and document the reason why the FDA-approved version is not appropriate.

In 2012, the FDA stated: "One factor that the agency considers in determining whether a drug may be compounded is whether the prescribing practitioner has determined that a compounded product is necessary for the particular patient and would provide a significant difference for the patient, as compared with the FDA-approved commercially available drug product" [34]. One might contend that cost constitutes a significant difference; however, the Pharmacy Compounding Accreditation Board Principles of Compounding states, "Price differences are not a 'significant' difference to justify compounding" [54].

Prescribing a compounded drug may expose providers to liability if a patient has a negative outcome, especially if a suitable FDA-approved product was available [3, 5557]. In the recent meningitis outbreak, a number of clinics, hospitals, and physicians have been named as defendants in lawsuits, along with the compounding pharmacy that prepared the contaminated drug. The American Society of Retina Specialists cautioned its members in 2012 to consider liability concerns when obtaining medications from compounding pharmacies [58]. Should a claim arise, medical malpractice insurance may exclude coverage if non-FDA approved drugs and procedures were used [59].

\section{Conclusion}

While drugs manufactured and tested in accordance with GMP regulations cannot be guaranteed to always be free of quality problems, the probability that FDA-approved drugs will consistently meet required quality standards is higher than it is for compounded drugs. Traditional pharmacy compounding provides an important therapeutic option to allow for the creation of individualized drug preparations when a patient's unique medical needs cannot be met with a commercially available drug. Examples include making dosage forms or strengths that are not commercially available or the removal of certain allergenic ingredients. In such cases, the option of prescribing compounded drugs should remain available for physicians. In traditional compounding, if a preparation error occurs, it would only affect a limited number of patients. Conversely, when pharmacy compounding is done at a large scale in uninspected facilities, using non-validated processes and ingredients of varying quality, an error could potentially affect a large population of patients. GMPs were established by the FDA to reduce the level of risk inherent in the large-scale production of drugs. A comprehensive body of regulations governing every aspect of drug manufacture and testing-enforced through regular FDA inspectionsis required to achieve consistent high quality. Setting aside these controls and creating a new class of pharmaceutical manufacturing, done without FDA oversight, is not in the best interests of patients.

Acknowledgements Jennifer Gudeman, Michael Jozwiakowski, and John Chollet are employees of Ther-Rx Corporation, which markets FDA-approved pharmaceuticals. Dr. Randell participated in a Ther-Rx Clinical Advisory Board meeting, for which he was compensated as a paid advisor. The authors have no other conflicts of interest that are directly relevant to the content of this article.

Open Access This article is distributed under the terms of the Creative Commons Attribution Noncommercial License which permits any noncommercial use, distribution, and reproduction in any medium, provided the original author(s) and the source are credited.

\section{References}

1. Galson SK. Federal and State Role in Pharmacy Compounding and Reconstitution: Exploring the Right Mix to Protect Patients. Hearing on Oversight Before the Senate Comm. on Health, Education, Labor, \& Pensions, 108th Cong. 2003. http://www. fda.gov/NewsEvents/Testimony/ucm115010.htm. Accessed Sept 2012.

2. United States Food and Drug Administration. The special risks of pharmacy compounding. 2012. http://www.fda.gov/ForConsumers/ ConsumerUpdates/ucm107836.htm. Accessed Sept 2012.

3. Sellers S, Utian WH. Pharmacy compounding primer for physicians: prescriber beware. Drugs. 2012;72(16):2043-50.

4. Information Update on 17a-Hydroxyprogesterone Caproate (17P) from The American College of Obstetricians and Gynecologists and The Society for Maternal-Fetal Medicine-13 October 2011. http://www.acog.org/ /media/Announcements/20111013 MakenaLtr.pdf. Accessed Apr 2012.

5. Wilson LE, Blythe D, Sharfstein JM. Fungal meningitis from injection of contaminated steroids: a compounding problem. JAMA. 2012;308(23):2461-2.

6. United States Food and Drug Administration. CFR-Code of Federal Regulations Title 21: Part 211 Current Good Manufacturing Practice for Finished Pharmaceuticals. 2012. http://www. accessdata.fda.gov/scripts/cdrh/cfdocs/cfcfr/CFRSearch.cfm? CFRPart=211. Accessed Aug 2012.

7. National Association of Boards of Pharmacy. Model Pharmacy Act/Rules. 2012. http://www.nabp.net/government-affairs/modelpharmacy-act-rules. Accessed Jan 2013.

8. Boodoo JM. Compounding problems and compounding confusion: federal regulation of compounded drug products and the FDAMA circuit split. Am J Law Med. 2010;36(1):220-47. 
9. United States Food and Drug Administration. FDA Talk Paper: FDA Warns Against Women Using Unapproved Drug, Domperidone, to Increase Milk Production. 2004. http://www.fda. gov/Drugs/DrugSafety/InformationbyDrugClass/ucm173886.htm. Accessed July 2012.

10. United States Food and Drug Administration. Updated FDA Statement on Compounded Versions of hydroxyprogesterone caproate (the active ingredient in Makena). 2012. http://www. fda.gov/NewsEvents/Newsroom/PressAnnouncements/ucm308546. htm. Accessed July 2012.

11. Draper R. The Toxic Pharmacist. 2003. http://www.nytimes.com/ 2003/06/08/magazine/the-toxic-pharmacist.html?pagewanted= all\&src=pm. Accessed July 2012.

12. Kastango E. Quality-control analytical methods: USP chapter $\langle 797\rangle$ compounded sterile preparations sterility requirements and their relationship to beyond-use dating. Int $\mathrm{J}$ Pharm Compd. 2004;8(5):393-7.

13. Pharmaceutical compounding-sterile preparations (general chapter 797). United States Pharmacopeia 35-National Formulary 30. Rockville: United States Pharmacopeial Convention; 2012.

14. Sterility Tests (general chapter 71). United States Pharmacopeia 35-National Formulary 30. Rockville: United States Pharmacopeial Convention; 2012.

15. National Association of Boards of Pharmacy Model Pharmacy Act/Rules Page 207. 2012. http://www.nabp.net/governmentaffairs/model-actrules/. Accessed Apr 2012.

16. Texas State Board of Pharmacy, Business Meeting Minutes, February 9-10, 2010, Proposal of Rules, Rules Concerning Use of Sterile Gloves and Sterile Alcohol in Pharmacies Compounding Sterile Preparations (\$291.133]. 2010. http://www. tsbp.state.tx.us/files_pdf/min2_2010.pdf. Accessed Nov 2012.

17. United States Food and Drug Administration. Meds IV pharmacy, IV compounded products recall: outbreak of Serratia marcescens bacteremia in Alabama hospitals. March 30, 2011. 2011. http:// www.fda.gov/Safety/MedWatch/SafetyInformation/SafetyAlerts forHumanMedicalProducts/ucm249099.htm. Accessed Aug 2012.

18. Tainted TPN Cases Put Focus on $\langle 797\rangle$ Rules, Pharmacy Practice News, June 2011, Volume 38. 2011. http://issuu.com/mcmahon group/docs/ppn0611_de. Accessed Nov 2012.

19. Institute for Safe Medical Practices Safety Alert. TPN-related deaths call for FDA guidance and pharmacy board oversight of USP chapter $\langle 797\rangle$. 2011. http://www.ismp.org/newsletters/ acutecare/articles/20110407.asp. Accessed July 2012.

20. Fricker MP, Trissel LA, Rich DS. Turning a new chapter on IV drug compounding safety: USP/NF chapter $\langle 797\rangle$. Hosp Pharm. 2004;9:899-920.

21. ACOG Committee opinion no. 532: compounded bioidentical menopausal hormone therapy. Obstet Gynecol. 2012;120 (2 Pt 1):411-5.

22. Newton D, Trissel L. A primer on USP chapter $\langle 797\rangle$ "pharmaceutical compounding - sterile preparations", and USP process for drug and practice standards. Int $\mathrm{J}$ Pharm Compd. 2004;8(4):251-63.

23. Health Canada: Health Products and Food Branch Inspectorate. Policy on manufacturing and compounding drug products in Canada POL-0051. 2009. http://www.hc-sc.gc.ca/dhp-mps/alt_ formats/hpfb-dgpsa/pdf/compli-conform/pol_0051-eng.pdf. Accessed Jan 2013.

24. United States Food and Drug Administration. Limited FDA Survey of Compounded Drug Products. 2001. http://www.fda. gov/Drugs/GuidanceComplianceRegulatoryInformation/Pharmacy Compounding/ucm155725.htm. Accessed Mar 2013.

25. US Food and Drug Administration. Pharmacy Compounding. 2006 Limited FDA Survey of Compounded Drug Products. 2012. http://www.fda.gov/Drugs/GuidanceComplianceRegulatory
Information/PharmacyCompounding/ucm204237.htm. Accessed Sept 2012

26. Missouri Board of Pharmacy Compounding Report. FY20062009. http://pr.mo.gov/pharmacists-compounding.asp. Accessed Mar 2013.

27. Sasich LD, Sukkari SR. Unknown risks of pharmacy-compounded drugs. J Am Osteopath Assoc. 2008;108(2):86.

28. Texas State Board of Pharmacy, Business Meeting Minutes, November 9, 2010, Report on TSBP Sampling of Compounded Products, Tab 24. 2010. http://www.tsbp.state.tx.us/files_pdf/BN/ Nov10/Additions/Tab24_Compounded\%20Sample\%20Testing. pdf. Accessed Nov 2012.

29. Azarnoff DL, Lee JC, Lee C, et al. Quality of extemporaneously compounded nitroglycerin ointment. Dis Colon Rectum. 2007;50(4):509-16.

30. Green DM, Jones AC, Brain KR. Content variability of active drug substance in compounded oral 3,4-diaminopyridine products. J Clin Pharm Ther. 2012;37(1):53-7.

31. Goldman MP. Sodium tetradecyl sulfate for sclerotherapy treatment of veins: is compounding pharmacy solution safe? Dermatol Surg. 2004;30(12 Pt 1):1454-6; discussion 1456.

32. Mahaguna V, McDermott JM, Zhang F, et al. Investigation of product quality between extemporaneously compounded progesterone vaginal suppositories and an approved progesterone vaginal gel. Drug Dev Ind Pharm. 2004;30(10):1069-78.

33. Chollet JL, Jozwiakowski MJ. Quality investigation of hydroxyprogesterone caproate active pharmaceutical ingredient and injection. Drug Dev Ind Pharm. 2012;38(5):540-9.

34. United States Food and Drug Adminstration. Questions and Answers on Updated FDA Statement on Compounded Versions of hydroxyprogesterone caproate (the active ingredient in Makena). 2012. http://www.fda.gov/newsevents/newsroom/press announcements/ucm310215.htm. Accessed Mar 2013.

35. Heinrich J. GAO testimony: Federal and State Role in Pharmacy Compounding and Reconstitution: Exploring the Right Mix to Protect Patients: Hearing on Oversight Before the Senate Comm. on Health, Education, Labor, \& Pensions, 108th Cong. 2003. http://gao.gov/assets/120/110456.pdf. Accessed Mar 2013.

36. Civen R, Vugia DJ, Alexander R, et al. Outbreak of Serratia marcescens infections following injection of betamethasone compounded at a community pharmacy. Clin Infect Dis Off Publ Infect Dis Soc Am. 2006;43(7):831-7.

37. Kato Z, Nakamura M, Yamagishi Y, et al. Pediatric thioridazine poisoning as a result of a pharmacy compounding error. Pediatr Rep. 2009;1(1):e9.

38. Romano MJ, Dinh A. A 1000-fold overdose of clonidine caused by a compounding error in a 5-year-old child with attentiondeficit/hyperactivity disorder. Pediatrics. 2001;108(2)471-2.

39. Sunenshine R, Schultz M, Lawrence MG, et al. An outbreak of postoperative gram-negative bacterial endophthalmitis associated with contaminated trypan blue ophthalmic solution. Clin Infect Dis Off Publ Infect Dis Soc Am. 2009;48(11):1580-3.

40. Suchard JR, Graeme KA. Pediatric clonidine poisoning as a result of pharmacy compounding error. Pediatr Emerg Care. 2002; 18(4):295-6.

41. Gershman MD, Kennedy DJ, Noble-Wang J, et al. Multistate outbreak of Pseudomonas fluorescens bloodstream infection after exposure to contaminated heparinized saline flush prepared by a compounding pharmacy. Clin Infect Dis Off Publ Infect Dis Soc Am. 2008;47(11):1372-9.

42. Schwam E. Severe accidental overdose of 4-aminopyridine due to a compounding pharmacy error. J Emerg Med. 2011;41(1):51-4.

43. McCoy KS. Compounded colistimethate as possible cause of fatal acute respiratory distress syndrome. N Engl J Med. 2007; 357(22):2310-1. 
44. Held MR, Begier EM, Beardsley DS, et al. Life-threatening sepsis caused by Burkholderia cepacia from contaminated intravenous flush solutions prepared by a compounding pharmacy in another state. Pediatrics. 2006;118(1):e212-5.

45. FDA Alerts Health Care Professionals of Infection Risk from Repackaged Avastin Intravitreal Injections. 2011. http://www. fda.gov/Drugs/DrugSafety/ucm270296.htm. Accessed Mar 2013.

46. Exophiala infection from contaminated injectable steroids prepared by a compounding pharmacy-United States, July-November 2002. MMWR Morb Mortal Wkly Rep. 2002;51(49):1109-12.

47. Deaths from intravenous colchicine resulting from a compounding pharmacy error-Oregon and Washington, 2007. MMWR Morb Mortal Wkly Rep. 2007;56(40):1050-2.

48. Moberg-Wolff E. Potential clinical impact of compounded versus noncompounded intrathecal baclofen. Arch Phys Med Rehabil. 2009;90(11):1815-20.

49. Pollack A. Avastin injections are reported to cause blindness. 2011. http://www.nytimes.com/2011/08/31/health/31drug.html?ref= avastindrug. Accessed Mar 2013.

50. Pollack A. Five More Reports of Avastin Injections Causing Blindness. 2011. http://www.nytimes.com/2011/09/02/business/ more-reports-of-avastin-causing-blindness.html?_r=1\&ref= avastindrug. Accessed Mar 2013.

51. Centers for Disease Control and Prevention. Multistate Fungal Meningitis Outbreak Investigation: Laboratory Testing and Results from the Outbreak. 2012. http://www.cdc.gov/HAI/ outbreaks/laboratory/lab_testing_results.html\#labresults. Accessed Nov 2012.
52. Food and Drug Administration Inspectional Observations (Form 483) New England Compounding Center issued October 26th, 2012. 2012. http://www.fda.gov/downloads/AboutFDA/Centers Offices/OfficeofGlobalRegulatoryOperationsandPolicy/ORA/ ORAElectronicReadingRoom/UCM325980.pdf. Accessed Nov 2012.

53. Kastango E. The cost of quality in pharmacy. Int $\mathrm{J}$ Pharm Compd. 2002;6(6):404-7.

54. Pharmacy Compounding Accreditation Board: $\mathrm{PCAB}^{\mathrm{TM}}$ Principles of Compounding. 2012. https://secure.pcab.info/about/ downloads/principles-of-compounding.pdf. Accessed Sept 2012.

55. Mckenna KJ. Compounded sclerosing agents: risks and consequences. Vein Mag. 2008;1(2).

56. Patel Y, Rumore MM. Hydroxyprogesterone caproate injection (Makena) one year later: to compound or not to compound that is the question. P T. 2012;37(7):405-11.

57. Gallegos A. Physicians entangled in tainted drugs lawsuits. 2013. http://www.amednews.com/article/20130211/profession/1302199 77/2/. Accessed Mar 2013.

58. Compounding Pharmacies-What Every Retina Specialist Needs to Know. 2012. http://www.asrs.org/education/compoundingpharmacies-/background. Accessed Nov 2012.

59. Kabnick LS. Compounded Sclerosants And Foam: What Should You Know About This Controversial Area? Legal Guidelines for Use of Polidocanol and Sodium Tetradecyl Sulfate for Sclerotherapy. Veith Symposium; 19-23 Nov 2008; New York. 